\title{
Application of UAV 3D Tilt Photography Technology in Mine Monitoring
}

\section{Chen Lin}

Henan Nonferrous Surveying and Mapping Co., Ltd., Anyang, Henan, 450016, China

\begin{abstract}
Three-dimensional tilt photography technology of UAV is a new technology for the development of new era. It not only successfully solves the shortcomings of traditional measurement technology, but also improves the resolution, accuracy and imaging effect. Therefore, this paper will take UAV three-dimensional tilt photography technology as the main object, and analyze its monitoring application in mine engineering, so as to improve the market value of the technology, highlight the application characteristics of the technology, and promote the development of the technology in environmental protection and on-site supervision of mine engineering.
\end{abstract}

\section{Keywords}

UAV; 3D oblique photography; mine engineering

\section{无人机三维倾斜摄影技术在矿山监测中的应用}

林琛

河南省有色测绘有限公司，中国・河南安阳 450016

\section{摘 要}

无人机三维倾斜摄影技术是新时代发展所需的新型技术, 其不仅成功解决了传统测量技术的缺点, 同时还提高了分辨率、准 确度和成像效果。因此, 论文以无人机三维倾斜摄影技术为主要对象, 针对其在矿山工程中的监测应用进行分析, 进而提高 该技术的市场价值，突出该技术的应用特点，推动该技术在矿山工程的环境保护和现场监管等方面的发展。

\section{关键词}

无人机；三维倾斜摄影技术；矿山工程

\section{1 引言}

矿产资源作为生产活动中的重要的投入要素, 在中国现 代化和工业化发展过程中起着很重要的战略作用。然而, 长 期以来在对矿产资源的开发和利用过程中, 存在着诸多如安 全、管理、环境和技术等方面的问题 ${ }^{\left[{ }^{1}\right.}$ 。由于开采技术的落 后和环境保护规划的缺位, 矿产资源的开发必然会给环境带 来一定的破坏, 如开发间接导致的泥石流, 固体废弃物乱堆等。

从企业角度, 矿山测绘通过为矿山开发提供技术和管理 指导从而促进矿山资源开采效率, 增强企业经济效益。从政 府监管角度, 监管部门需要对矿产企业是否存在越界开发、 违法开采、破坏环境等方式进行定期监管, 面临着劳动投入、 监测周期、监管精度等方面的选择 ${ }^{[2]}$ 。无人机摄影技术拥有 快速、高效、高精度等优势, 可为矿区监管提供有力技术支撑。
已经广泛应用于土地变化、作物估产、野外作业、生态环境 评价等领域。利用无人机等遥感技术实现对地表的不间断监 测, 有助于加强对矿山的地质背景情况了解, 如地层岩性、 地质构造等, 促进对占用土地、矿山地质灾害分布情况等开 发利用的管理。

\section{2 无人机倾斜摄影概述}

无人机是指具有自动驾驶能力的飞行器, 一般是通过无 线遥感设备进行控制, 对规定区域进行信息采集并传回, 由 地面接收设备进行信息接收, 通过对信息的分析、确认、篮选, 最终达到收集所测区域的空间的、全方位的信息。倾斜摄影 技术是通过一个垂直、四个倾斜、五个不同的视角同步采集 影像的，从而获得更加丰富、清晰的资料。该技术是国际测 绘领域发展的一项新技术, 其打破了传统的拍摄局限, 由一 飞行平台和多台传感器完成了影像采集的工作。倾斜摄影技 
术不仅可以真实地反映所测区域的地物、地貌, 保障所测事 物信息的精确度, 而且能够在定位、建模等技术基础上构建 三维城市模型, 将给人们带来更加清晰、直观的影像。无人 机倾斜摄影测量技术能够记录摄影瞬间所测区域地表任意角 度的影像信息和三维坐标信息，具有高精度、高分辨率、高 效的特点, 能够反映所测区域的地形地物和周边环境 的实际 情况, 在测量工作中具有不可替代的优势 ${ }^{[3]}$ 。

\section{3 无人机三维摄影技术的基本内容}

无人机通常指的是无人驾驶的、具有自动驾驶能力的飞 机或其他飞行器, 其利用无线遥感设备进行控制, 逐步实现 对相关区域的观测和信息采集, 并将采集的信息及时传回地 面接收设备, 再结合一定的数据处理进行信息的确认和篮选, 进而实现全方位的、空间上的地质地形等的信息采集 ${ }^{[4]}$ 。因此, 无人机具备了灵活、轻便、经济效益高、造价成本低、可利 用条件好等多种优势, 实现了在航拍、矿山、地质地形、农业、 河流、灾害区域、野生动物区、雨林区及传染病的监控方面 的高效利用。

在无人机的系统组成方面, 通常包括: (1)飞行平台, 主 要负责飞行及相关数据信息的观测、采集和监控: (2)控制系统, 通常以遥感技术为基础, 结合飞行控制技术和设备管理技术, 在自驾、定位等的配合下, 实现无人机的整体控制; (3)摄影 传感系统, 主要是一种摄影传感器, 在无人机的飞行过程中, 通过对快门进行定点曝光控制, 对焦固定和相机锁定等技术 来实现三维的地面信息摄影; (4)地面接收系统, 主要用于接 收飞行器采集到的信息, 并对其进行数据处理、分类、归纳, 进而得出所观测区域的具体信息, 保障整个过程的信息准确、 真实、可靠。

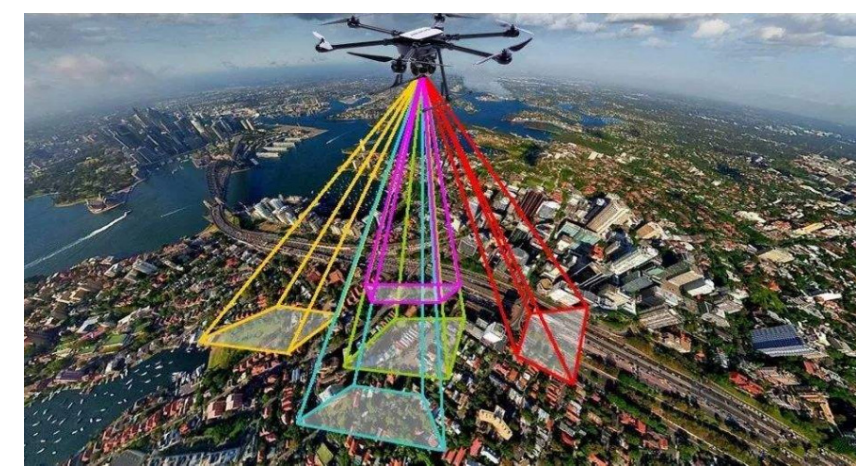

图 1 无人机三维倾斜摄影工作示意图

倾斜摄影技术是在高新技术的基础上逐步发展而来的新
型摄影测量技术，其在垂直角度、四个倾斜角度等方位都能 实现同步的信息采集，尤其是在建筑物的观测过程中，极大 程度地增加了观测数据的真实性、客观性、可靠性 ${ }^{[5]}$ 。无人 机三维倾斜摄影工作示意图如图 1 所示。

鉴于以上情况，市场上流通的无人机在矿山工程的观测 过程中基本具备了以下几种优势：(1)能够实现全方位、全过 程的实时监控, 保障了整个矿山工程开采的安全性; (2)能够 实现垂直、倾斜等多个观测角度的信息采集, 能准确的反应 所测区域的具体情况; (3)能够保障观测区域的信息真实性、 清晰性, 纹理的可观测性, 数据的可信赖性; (4)能够实现倾 斜摄影影像中的单张影像的量测; (5)观测数据的量小, 传输 过程简单, 有助于数据共享 ${ }^{\left[{ }^{[}\right]}$

\section{4 无人机倾斜摄影技术}

倾斜摄影技术：主要包括倾斜影像数据获取和倾斜影像 数据处理。倾斜影像数据获取是通过在同一飞行平台上搭 载 多台传感器, 实现从多个角度获取地形地貌信息, 通常采用 5 台照相机, 垂直地面的镜头所拍摄的成为正片, 其他与地 面成一定夹角的为斜片。倾斜影像数据处理通过将正片与斜 片进行多视角联合平差、影像密集匹配、3D纹理映射等过程, 生成基 “全要素、全纹理” 的实景三维模型。无人机倾斜摄 影测量系统, 主要由无人机系统、任务载荷系统、数据处理 系统等部分组成。

\section{1 无人机系统}

作为遥感航测飞行平台, 在无人机系统需要搭载和安装 一定重量和体积的任务设备; 首先应具备足够的安全性和可 靠性。同时航高和飞行航迹控制精度要满足摄影测量的基 本 要求。根据项目的具体环境, 作业区域的范围, 选择具体规 格和型号的航摄无人机。

\section{2 任务载荷系统}

主要包括倾斜摄影相机、同步控制装置、稳定云台等。 其中，倾斜相机及多角度同步控制装置用于影像同步获取， 是必须配备的构件; 稳定云台、位置与姿态采集系统的配备 可提高影像获取精度, 提升三维建模的效果 ${ }^{[7]}$ 。

\section{3 数据处理系统}

数据处理系统是也倾斜摄影测量的重要组成部分, 通常 采用三分布式计算机制, 而具备海量数据处理能力, 通过倾 
斜摄影三维软件, 利用连续的二维图像, 就能还原出实景三 维模型。

\section{5 无人机三维倾斜摄影技术在矿山监测中的应 用分析}

\section{1 工作流程}

无人机倾斜摄影技术可以完成矿山地形的多角度拍摄, 将地形的真实情况记录下来, 并能够完整、清晰地显示信息。 该技术通过大规模的高空拍摄, 收集到矿测绘。

山地质及纹理的数据信息, 并以三维立体模型的方式展 现出来。主要的工作包括以下几个方面: (1)前期的考察调研 工作。为了保障测量数据的有效性, 在进行无人机航拍前应 进行一定的准备工作, 即矿山的前期考察调研工作。通过该 工 作对矿山的具体情况进行了解, 主要内容包括: 确定矿山 拍摄范围、确定并调整摄影影像的分辨率等。(2)确定拍摄航线。 完成前期的调研工作后, 应及时整理和研究获得的数据信息。 通过一系列的整合工作, 将所有信息进行数字化, 结合无人 机的控制软件, 做好影像分辨率的调整, 同时加入航拍高度 画面的各种参数，从而规划和确定航拍的路线。(3)内外业的 航拍与处理阶段。在航拍前, 要做好飞行平台的搭建 和各个 传感器的调整工作, 使得两者能够统一、有效地进行工作。 在无人机倾斜技术拍摄的过程中, 要做好矿山各种相 关数据 信息的记录工作, 并统一的进行整理、分析 ${ }^{[8]}$ 。在完成矿山 的无人机倾斜摄影工作后, 应由专业人员借助一些系统软件, 分析和处理所拍摄的信息, 最终建立一个三维立体的模型图 像。就无人机倾斜摄影技术而言，其不仅可以实现拍摄地现 场信息的实时传送, 还能对矿山的地理位置、有关数据进行 准确的展示, 从而保障矿山地形测绘信息的完整性、可靠性。

(4)分析和评价测量结果。检查点的结果是否精准, 影响着矿 山地形测量结果的评定。因此, 要严格检测矿山地形的检查点, 检测项目的合格程度和完成程度。尤其是矿山地形拍摄画面 是否存在明显的拼接痕迹方面进行详细检查, 在一定程度上 确保摄影资料的准确性。

\section{2 矿山开采区域实时监测}

一般情况下, 无人机三维倾斜摄影技术是在露天情况下 实施监测矿山的开采情况。在实际的监测过程中, 无人机三 维倾斜摄影技术能够呈现高分率的图像和数据信息, 真实反
映矿山的开采情况 ${ }^{[9]}$ 。在获取的图 像中, 能够体现出矿山开 采区域的地形地貌，通过该技术生成 的三维立体模型能够还 原矿山的情况, 反映出开采进度及步骤, 在一定程度上降低 了矿山测量工作人员的工作任务和监测管理人员的工作量。

\section{3 应用成果分析}

在矿山工程的开采监测工作中, 无人机三维倾斜摄影记 得胡的应用成果有: (1)高分辨率的 DOIM 数据的获取, 高分 辨率的三维倾斜立体数据模型的建立, 实现了整个矿山工程 观测区域的实时监测, 保障了整个工程的安全开展; (2)实现 了 倾斜立体模型的多方位测量, 土方量的实际测量, 提高了 矿山观测区域的直观性、准确性、及时性和有效性; 3)真实 反映矿山工程开采情况, 提高了现场管控效率, 降低了人力 消耗, 保障了开采的生产调度, 工程进度、以及各项辅助工 程 (排水疏 干工程、环境保护工程等) 的开展的高效、高质 和高量。

\section{6 无人机倾斜摄影测量技术的其他应用及实例 分析}

\section{1 无人机倾斜摄影测量技术的其他应用}

由于无人机倾斜测量技术及三维实景模型具有一定的优 势, 使其在测绘方面得到了广泛的应用。该技术的应用主要 包括以下几个方面: 一是城市规划方面, 无人机倾斜测绘技 术形成的城市三维实景模型能够实现城市景观、景区等方面 的虚拟呈现, 通过网络能够对城市整体进行汶览, 为城市规 划工作提供了一定的帮助; 二是矿山监测方面, 无人机倾斜 摄影测量可以灵活快速地获取所测区域影像, 完成地形图的 生产、更新, 可进行竣工测量等 ${ }^{[10]}$; 三是电力规划方面, 电 力是中国经济发展的重要保障, 通过无人机倾斜摄影测量技 术能够快速实现线路走廊的三维环境建立, 从而对电力线路 带状区域的相关信息进行准确获取; 四是该技术还为国土资 源、公安、环保等各行各业提供基于三维虚拟现实的高精度 三维地理信息服务。

\section{2 无人机三维倾斜技术在矿山监测中的应用实例 分析}

待测区域为甘肃酒泉铁铜多金属矿区的一部分, 利用 无人机三维倾斜技术进行矿山监测, 主要进行了以下几方面 内容: 确定拍摄范围、航线设计、控制点布设、影像数据处 
理。航拍的相关参数为: 飞行区域为 $10 \mathrm{~m} 2$, 飞行高度为航高 $500 \mathrm{~m}$ ，使用的相机为 EOS 5D MarkII。

航线设计：根据 Kappa 角的变化对其进行航带划分, 规 划出的航线为,垂直摄影 11 条航线、倾斜角度摄影 22 条航线。 控制点布设：根据航线的划分和矿区的实际情况，对其周围 进行了控制点布设, 大概布设了 300 个控制点。影像数据处理. 该过程的流程为影像自动匹配 $\rightarrow$ 地面控制点量测 $\rightarrow$ 影像匹配 加密提取 DEM $\rightarrow$ DOM 与全景图制作。通过无人机三维倾 斜技术能够对矿山的情况进行实时的监测, 监测矿区的地形、 地质，对地质灾害防控和环境保护方面具有重要意义。

\section{7 结语}

总之, 将无人机三维倾斜摄影技术应用到矿山监测中, 能够保障矿区数据的可靠性、信息真实性等; 实现矿山开采 全过程、全方位的实时监控, 保障了整个矿山工程的安全性。 该技术的测量优势、三维模型优势等, 使其应用到各个领域, 进一步推动了该技术的发展。

无人机三维倾斜摄影技术作为现代化的测绘技术，其不 仅涵盖了信息化、数字化的技术能力, 还在极大程度上提升 了经济适用性、测绘高效性、数据真实性、测量有效性等方 面的能力，同时结合其在垂直方向和四个倾斜角度上的测量 优势, 以及真实还原地质地形的特点, 将大大提升该技术的
应用市场，将继续推动该技术的发展。

\section{参考文献}

[1] 张军, 高洁纯. 无人机三维倾斜摄影技术在矿山监测中的应用 [J]. 几世界有色金属, 2018(19): 17-18.

[2] 涂勇, 万昕. 无人机三维倾斜摄影技术在露天矿山监测中的实践 与探索 [J]. 世界有色金属, 2018(09):22-23.

[3] 韦小儒. 无人机倾斜摄影测量在露天矿山监测中的应用研究 [J]. 世界有色金属, 2018(07):21-22

[4] 张玉侠, 兰鹏涛, 金元春, 等. 无人机三维倾斜摄影技术在露天矿 山监测中的实践与探索 [J]. 测绘通报 ,2017(S1):114-116.

[5] 郭岗, 王春涛, 赵元务. 无像控无人机倾斜摄影测量在农村地籍测 量中的应用 [J]. 测绘与空间地理信息, 2019(04):216-218.

[6] 杜甘霖, 叶茂, 刘玉珠, 等.露天矿山监管中的无人机测绘技术应 用研究 [J]. 中国矿业, 2019(04):111- 114

[7] 杨丰宾, 许建民. 无人机三维倾斜摄影技术在矿山监测中的应用 [J]. 世界有色金属, 2018(24).

[8] 胡月. 无人机三维倾斜摄影技术在矿山监测中的应用 [J]. 世界有 色金属, 2019(10)

[9] 李淑军. 无人机三维倾斜摄影技术在露天矿山监测中的实践与探 索 [J]. 世界有色金属, 2019(7):194-194.

[10] 李少军. 浅析无人机倾斜摄影测量方法在矿山中的应用 [J]. 中国 金属通报, 2018(05):64-65. 